dup7q11.23, located in the long q arm of chromosome 7, at the region commonly deleted in WBS. The characteristic facial phenotype is also shared with a previously described syndrome of delay in expressive language and supernumerary ring chromosome 7. This consists of a high, broad nose, posteriorly rotated ears, high-arched palate, and short philtrum. These facies coupled with expressive language delay should prompt genetic testing for duplication of the WBS region.

An editorial perspective "On genes, speech, and language" (Fisher SE. N Engl J Med Oct 20, 2005;353:1655-1657) refers to a specific gene, FOXP2, located in chromosome 7q31, implicated previously in verbal dyspraxia and orofacial dyspraxia (Macdermot KD et al. Am J Hum Genet 2005;76:1074-1080; cited by Somerville et al). The above study that identifies a second locus on chromosome 7 associated with language delay and oromotor dyspraxia emphasizes the potential of using molecular diagnostics for early identification of children at increased risk of language and speech impairment.

\title{
FOCAL BRAIN LESIONS AND LANGUAGE DEVELOPMENT
}

The effects of congenital, unilateral, focal brain lesions on development and hemispheric lateralization of language were investigated in 24 preschool children with hemiplegia followed longitudinally at Stella Marios Scientific Institute, Pisa, Italy. Twelve had left hemisphere damage (LHD) and 12 right hemisphere damage (RHD). Linguistic assessment at 2 and 3 years of life showed early left-side specificity for language. As shown by the Fused Dichotic Words Listening Test, a shift of language lateralization to the opposite hemisphere occurred in all 12 children with LHD and in 4 of 12 with RHD. The most atypical lateralization coefficients (values more than 2 standard deviations from the mean of a normal sample) were associated with: 1) delay in lexical and grammatical development, especially after LHD; 2) cortical-subcortical-periventricular lesions more than solely periventricular damage; and 3) larger rather than smaller lesions. A shift of language functions to alternative brain regions, as a result of focal brain damage, is associated with a slow rate of language development. EEG abnormalities alone did not affect language development, but children with seizures were less advanced in expressive grammar and had lower cognitive scores than those with EEG abnormalities but no seizures $(p<0.05)$. (Chilosi AM, Pecini C, Cipriani $\mathrm{P}$ et al. Atypical language lateralization and early linguistic development in children with focal brain lesions. Dev Med Child Neurol November 2005;47:725-730). (Respond: G Cioni MD, Department of Developmental Neuroscience, IRCCS Stella Maris-University of Pisa, Via dei Giacinti 2, 56018 Calambrone, Pisa, Italy).

COMMENT. In children with congenital, focal brain damage and resulting atypical language lateralization, the risk of impaired cognitive and language outcome is increased by large cortical-subcortical lesions of the left hemisphere. The occurrence of seizures accentuates the impairment of language.

Abnormal speech sound representation in persistent developmental stuttering (PDS) is demonstrated in a comparison of mismatch negativity (MMN) potentials elicited in PDS subjects and paired fluent control subjects. (Corbera S et al. Neurology October (2 of 2) $2005 ; 65: 1246-1252)$. MMN is a cognitive evoked potential elicited to unexpected auditory stimuli. An abnormal speech sound representation in the auditory cortex may underlie PDS. 